\title{
¿Es el derecho internacional de los derechos humanos una herramienta efectiva para impulsar el aborto legal a nivel nacional? Un análisis del caso argentino
}

\author{
Is international human rights law an effective tool for \\ achieving legal abortion at the national level? \\ A study on the Argentine case
}

Resumen: Este artículo busca analizar la experiencia argentina en términos de legalización del aborto. Para lograrlo, este estudio presentará y analizará críticamente los antecedentes legales en Argentina respecto a la temática, refiriéndose a la normativa originaria, su aplicación y evolución. Se cubrirá el desarrollo legislativo a través del tiempo y se referirá puntualmente al tratamiento del proyecto de ley de aborto presentado en 2018, como parte de dicho proceso. Al mismo tiempo, la investigación abordará el rol y la participación de los movimientos sociales en relación con el aborto. Posteriormente, este estudio contrastará la experiencia localizada con el desarrollo macroscópico del Derecho Internacional

\footnotetext{
1 Luciana Wechselblatt es abogada, graduada de la Universidad de Buenos Aires (Argentina, 2015) y magíster en Derecho Internacional, con orientación en derechos humanos y protección de grupos vulnerables, del Instituto de Altos Estudios Internacionales y de Desarrollo de Ginebra (Suiza, 2018-2019). La autora agradece a la Dra. Neus Torbisco Casals por su valiosa guía y acompañamiento en la producción de este artículo.
} 
de los Derechos Humanos (DIDH) respecto al aborto y considerará su potencial impacto en el país analizado. Se pondrá atención especialmente a los instrumentos legales más recientes que refieren a la terminación del embarazo y su función en el contexto nacional. Finalmente, las dos secciones finales de este estudio proveerán algunas reflexiones respecto al rol del DIDH en esta temática, sus contribuciones y sus puntos débiles, en vistas a la falta de adopción del proyecto de ley de aborto en Argentina presentado en el año 2018.

Palabras claves: Aborto; Argentina; Movimientos sociales; Enfoque descendente; Enfoque ascendente.

Abstract: This article aims to analyze the Argentine experience on the legalization of abortion. To do so, this study will present and critically analyze the legal background in Argentina by first referring to the initial framework, its application, and how it evolved. It will cover the legal development over time and will refer to the treatment of the abortion bill in 2018 as part of this process. Alongside, the research will also address the role and participation of social movements concerning abortion. Afterwards, this study will contrast the localized experience with the macroscopic development of international human rights law (IHRL) as regards abortion and consider its potential impact on the country under analysis. Special attention will be given to the most recent international legal tools that refer to the termination of pregnancy and its function in the national context. In light of this, the last two sections of the study will provide some insights as regards the role of IHRL in this regard, its contributions and flaws, in view of lack of adoption of the bill in Argentina.

Keywords: Abortion; Argentina; Social movements; Top-down approach; Bottom-up approach. 


\section{Sumario}

\section{Introducción}

2. La experiencia nacional en Argentina

a) Contexto legal

b) 2018: El proyecto de ley de aborto

c) El debate

d) Últimos avances y consideraciones finales

3. El contraste de la experiencia nacional a la luz de los estándares internacionales

a) Panorama general

b) Caso de estudio de los órganos de tratados: El Comité de Derechos Humanos

c) Conclusión de la sección

\section{Reflexiones finales}

5. Referencias bibliográficas

\section{Introducción}

En la actualidad, nos encontramos ante un desarrollo vertiginoso de la visibilización y reclamo de los derechos de las mujeres, que se han tornado en una prioridad en las agendas de derechos humanos a nivel internacional y nacional. Recientemente, se han ido intensificando campañas masivas de sensibilización sobre necesidades y riesgos específicos que enfrentan las mujeres. Al momento, hay dos procesos que están teniendo lugar: la incorporación de la perspectiva de género -o gender mainstreaming - (ONU Mujeres), que promueve el uso transversal de esta perspectiva al analizar y promover todo tipo normas y políticas 
de derechos humanos; y los derechos específicos de género, ${ }^{2}$ que pertenecen concretamente a las mujeres ${ }^{3}$ como grupo. Uno de los derechos específicos de la mujer que se encuentra en debate es el derecho al acceso a un aborto legal, seguro y gratuito. ${ }^{4}$

Inserto en este contexto, el microcosmos de la experiencia argentina en miras a la legalización del aborto ofrece aportes muy enriquecedores por varias razones. Incluye la cuestión de la interacción entre la ley y la sociedad, ofrece una robusta presencia de los movimientos sociales y una posible contribución de elaboración legal con un enfoque ascendente (bottom-up approach). ${ }^{5}$ Asimismo, el análisis de la situación en Argentina también permite poner el foco en la experiencia nacional de un país cuya agenda de derechos humanos ejerce un rol influyente en la región latinoamericana, donde la liberalización del aborto es un gran reto.

\footnotetext{
2 Por ejemplo, algunas amenazas que específicamente afectan a las mujeres y niñas pueden ser mencionadas: la discriminación y violencia basada en sexo y género, el tráfico de personas, las prácticas y legislación relativa a la familia, que afectan desproporcionadamente mujeres y niñas, los estereotipos de género, entre otros. Se puede acceder a información adicional en "Women's Rights are Human Rights", texto publicado por la Oficina del Alto Comisionado para los Derechos Humanos, 2014, disponible en: https://www.ohchr.org/Documents/Publications/HR-PUB-14-2.pdf

${ }^{3} \mathrm{Al}$ referirse a las mujeres en el transcurso de este ensayo, se pretende abarcar también niñas y adolescentes, a menos que se haga una distinción al respecto.

${ }^{4}$ Una observación importante merece ser realizada: este artículo reconoce los derechos del colectivo LGBTIQ+, lo cual también implica que dar a luz es un atributo de cada cuerpo gestante, no solamente de las mujeres. Esto significa que otras identidades sexuales tales como personas trans y de género neutral también pueden concebir. Sin perjuicio de esto, y del hecho de que los derechos de las mujeres y del colectivo LGBTIQ+ son aliados, este estudio se enfocará en el aborto principalmente como un derecho de las mujeres. De la misma forma, este estudio pretende utilizar lenguaje inclusivo y evitar las generalizaciones en género masculino. Este tipo de fórmulas se utilizarán solamente cuando sean estrictamente necesarias y para facilitar la lectura.

${ }^{5}$ Esta posición refiere al poder de movimientos localizados y de comunidades en la elaboración de la ley. Algunos académicos sostienen que los derechos humanos emanan de los movimientos sociales y de las luchas políticas realizadas por personas "ordinarias". Más información en: Rajagopal, International law and social movements: Challenges to theorizing resistance (Columbia Journal of Transnational Law, 2002); Stammers, Human rights and social movements, (London: Pluto Press, 2009).
} 
En 2018 fue la primera vez que el proyecto de ley de aborto fue tratado en el Congreso argentino, luego de más de una década de presentaciones de proyectos. Tras un amplio debate, que involucró congresistas, personas expertas en la materia y representantes de la sociedad civil, la Cámara de Diputados aprobó el proyecto de ley por un apretado margen, mientras que la Cámara de Senadores lo rechazó, también por un margen estrecho (Honorable Senado de la Nación, 2018).

$\mathrm{Al}$ analizar el fracaso en la aprobación del proyecto de ley, este artículo pretende avocarse a, principal pero no únicamente, responder las siguientes preguntas: ¿Hasta qué punto la agenda de derechos humanos y los movimientos sociales proaborto pueden recurrir a los sistemas internacionales de protección y promoción de los derechos humanos como una fuerza que empuja hacia la aprobación de una ley nacional? ¿Cuáles son las contribuciones y posibles fallas del derecho internacional de los derechos humanos (DIDH)? ¿Debería este marco servir como una herramienta de cambio que promueva el aborto legal?

\section{La experiencia nacional en Argentina}

\section{a. Contexto legal}

\section{Contexto legal histórico}

En Argentina la regulación del aborto tiene lugar a través del Código Penal. El artículo 86, inciso $2^{6}$-que puede ser encontrado

\footnotetext{
${ }^{6}$ Código Penal, artículo 86: Incurrirán en las penas establecidas en el artículo anterior y sufrirán, además, inhabilitación especial por doble tiempo que el de la condena, los médicos, cirujanos, parteras o farmacéuticos que abusaren de su ciencia o arte para causar el aborto o cooperaren a causarlo. El aborto practicado por un médico diplomado con el consentimiento de la mujer encinta, no es punible:

$1^{\circ}$ Si se ha hecho con el fin de evitar un peligro para la vida o la salud de la madre y si este peligro no puede ser evitado por otros medios.
} 
bajo el título de "Crímenes contra la vida" - existe desde el año 1921 y prescribe casos específicos en que el aborto no es criminalizado: casos de violación y riesgo en la vida o la salud de la persona embarazada.

Sin embargo, tras su establecimiento, ningún servicio de salud fue puesto a disposición para que estos supuestos permisibles se encuentren disponibles en la práctica (Bergallo, 2014, 243). No solamente la falta de disponibilidad de servicios resultó un obstáculo de facto en el acceso al aborto bajo las causales aceptadas, sino que también se instalaron otras prácticas dañinas que en muchos casos daban cuenta de la falta de voluntad de las instituciones de salud para responder a este hecho, entre ellas: el abuso de la objeción de conciencia por parte de los trabajadores de la salud, el uso excesivo de la judicialización que buscaba dilatar el acceso por parte de las instituciones médicas, los requisitos dilatorios estipulados por los protocolos sanitarios, la discriminación de los sistemas de salud, los discursos moralizadores por parte de los agentes de la salud, los casos de violación del secreto profesional, la influencia de la iglesia en el gobierno nacional, provincial y local , entre otras (CELS, 2018a). Más aún, la existencia del riesgo de persecución penal para toda mujer buscando acceder al aborto mismo bajo las causales permitidas también disuadió a las mujeres de solicitarlo.?

Como resultado, Argentina se sumergió en una "prohibición informal” (Bergallo, 2014, 243) que subvirtió la regulación formal y estableció un "veto moral" (Bergallo, 2014, 246). No obstante, los abortos ocurrían a pesar de su criminalización, de la prohibición

\footnotetext{
$2^{\circ}$ Si el embarazo proviene de una violación o de un atentado al pudor cometido sobre una mujer idiota o demente. En este caso, el consentimiento de su representante legal deberá ser requerido para el aborto.

${ }^{7}$ Como será explicado más adelante, la existencia de este riesgo es variable. Mientras que en algunas provincias del país el riesgo resulta bajo y sumamente improbable, en otras provincias y regiones la persecución penal es un riego real para toda mujer que interrumpa su embarazo. Esto da cuenta de la realidad fragmentada que presenta la Argentina.
} 
moral y de la falta de infraestructura sanitaria correspondiente. En consecuencia, la situación prevaleciente derivó inevitablemente en que las mujeres tuvieran que recurrir a prácticas clandestinas para lograr su acceso (ELA, REDAAS, CEDES, 2018). Este contexto acarreó al menos tres consecuencias graves.

En primer lugar, ubicó el aborto en la reserva de la esfera privada - y fuera de la regulación pública. Esto implicó que el acceso de cada mujer dependiera de sus medios sociales, culturales y, sobre todo, económicos. Como consecuencia, una red de proveedores privados fue construida para ofrecer el acceso al aborto a las personas que pudieran costearlo. Esta red operaba en la clandestinidad, sin control regulatorio ni interferencia de las fuerzas de cumplimiento de la ley, en desconocimiento total de la razón por la cual se realizara el aborto. Este panorama también contribuyó a crear un incentivo económico para los trabajadores y trabajadoras de la salud y otros actores al interior del mercado ilegal, convirtiéndose en un obstáculo adicional para la legalización (Sedacca, 2017, 114).

Como resultado, en la práctica en Argentina sólo podrían acceder al aborto aquellas mujeres pertenecientes a un estatus socioeconómico-cultural ligado a la clase media y media alta, y aquellas que contaran con el acceso a "redes de rescate" (CELS, 2018b, 23). Estas redes eran esencialmente grupos militantes que acompañaban a las mujeres que no podían costear un aborto privado y las ayudaban a acceder con su apoyo (esta táctica será discutida con mayor detalle posteriormente).

En segundo lugar, toda mujer embarazada que quisiera terminar su embarazo producto de una violación aún corría el riesgo de ser castigada debido a que los supuestos aceptados por el Código Penal eran inaccesibles derivadas de las prácticas hospitalarias anteriormente mencionadas, que resultaban en obstáculos prácticos en el acceso. Por lo tanto, las mujeres quedaban confinadas a recurrir al aborto clandestino y esto, asimismo, producía un efecto disuasivo con relación a la denuncia de la violación. Este escenario también 
perpetuaba (y aún lo hace) la cultura de la violación debido a la falta de denuncia, la pérdida del estatus de víctima de violencia sexual contra la mujer ${ }^{8}$ y la consecuente impunidad del perpetrador.

En tercer lugar, este contexto podía (y aún puede) conducir a la extrema distorsión en la percepción del rol de las mujeres e incluso de las niñas en la sociedad, confinándolas a la mera función de madres y de proveedoras de cuidado, lo cual refleja claros estereotipos de género. ${ }^{9}$ Por ejemplo, en febrero de 2019 una niña de 11 años de la provincia de Tucumán, que fue violada por un familiar, solicitó la interrupción de su embarazado, en utilización de una de las causales reguladas por el Código Penal. Sin embargo, fue obligada a continuar su embarazo y llevar a cabo una cesárea para "salvar el feto" (Clarín, 2019a). Asimismo, el accionar mediático convalidó la percepción del rol de las niñas en la sociedad como madres. Así, el diario "La Nación" publicó un artículo de opinión, cuyo autor es desconocido, en el que refirió que para las mujeres de bajos recursos (y, según el autor, inclusive las niñas), dar a luz era una fuente de orgullo, aún si el hecho fue el producto de una violación sexual (La Nación, 2019). La situación fue descripta bajo la reprensible noción de "niñas madres", que quedó inserta en el título del artículo. Esta opinión escandalosa fue masivamente repudiada a través de una campaña en redes sociales conocida como \#niñasnomadres (Página 12, 2019) cuyo fin fue sensibilizar sobre las vulnerabilidades de las niñas y adolescentes ante

\footnotetext{
${ }^{8}$ Corte Suprema de Justicia de la Nación, 12 de marzo de 2012, F., A.L., Expediente Letra “F," No. 259, Libro XLVI, (Arg.). Considerando 16. Esta sentencia será explicada luego con mayor profundidad.

${ }^{9}$ El trabajo de Cecilia M. Hopp titulado Política criminal sobre el aborto: la sexualidad femenina en debate (Sistema Argentino de Información Jurídica - SAIJ, 2012) también hace una reflexión sobre el uso performativo del lenguaje y cómo la utilización del término "madre" en lugar de "persona gestante" en pasajes del - en ese entonces Código Civil, posee una carga simbólica que ubica a la mujer destinada a ese rol; Gabriela Irrazabal y Karina Felitti. El Caso Belén y las construcciones sobre el "no nacido" en Argentina. Aportes para la discusión (Rev. Bioética y Derecho no.43, Barcelona, 2018). Este artículo detalla en profundidad el impacto de la terminología utilizada por la jurisdicción tucumana en el conocido caso Belén. Disponible en: http://scielo.isciii.es/scielo.php?script=sci arttext\&pid=S1886-58872018000200005
} 
estas situaciones. En otras palabras, los movimientos sociales buscaron luchar contra estereotipos tales que retratan a las mujeres como proveedoras de cuidado y destinadas a la maternidad.

\section{Evolución del contexto legal}

A raíz de la reforma constitucional de 1994, el marco legal argentino alcanzó un nuevo estadio que implicó una nueva gama de compromisos en materia de derechos humanos. La incorporación de distintos tratados internacionales de derecho internacional de los derechos humanos (DIDH) con jerarquía constitucional hizo que los magistrados y magistradas pudieran aplicar el control de constitucionalidad $^{10}$ y de convencionalidad que los obliga a corroborar que las provisiones de dichos instrumentos estén siendo aplicadas y no contravenidas por leyes domésticas. Al realizar esta labor, el poder judicial pasó a ser el primer intérprete de las normas internacionales (Pittier, 2016) en el caso concreto que el juez o jueza deba decidir. ${ }^{11}$ En el país, esta función es efectuada de manera difusa por todos los jueces nacionales (en ausencia de un tribunal constitucional) incluso ex $_{\boldsymbol{x}}$ officio (sin la necesidad de una solicitud). Esta reforma normativa impactó en la temática dado que el ordenamiento internacional de derechos humanos pasó a utilizarse

\footnotetext{
${ }^{10}$ La Corte Suprema de Justicia de Argentina y los tribunales inferiores han realizado este tipo de control por décadas. Algunos casos remarcables de la Corte Suprema son: "Ekmekdjian c. Sofovich", sentencia del 7 de julio de 1992; "Acosta”, sentencia del 28 de diciembre de 1998; "Espósito Miguel Ángel s/incidente de prescripción de la acción penal promovido por su defensa", sentencia del 23 de diciembre 2004; "Mazzeo, Julio Lilio s/recurso de casación e inconstitucionalidad", sentencia del 13 de julio de 2007.

${ }^{11 S i}$ bien se pone el énfasis en el control judicial, se debe destacar que “...esta tarea no solamente deben llevarla a cabo los jueces sino también el Poder Ejecutivo y a la par el propio Parlamento, que tiene el deber de no dictar preceptos violatorios de los Tratados". Juan Carlos Hitters, Reforma de la Constitución Argentina de 1994 y los Tratados sobre Derechos Humanos a 20 años de su vigencia (2014, Universidad de Friburgo). Disponible en: http://perso.unifr.ch/derechopenal/assets/files/ obrasportales/op 20141108 03.pdf
} 
en este tipo de contextos, ofreciendo la chance de interpretar su impacto y alcance a través del control de convencionalidad.

En paralelo a los cambios normativos, los movimientos sociales desarrollaron distintas estrategias apuntando a encontrar diversas maneras de superar las dificultades legales y prácticas en el acceso legal al aborto y sus peores consecuencias, ya descritas anteriormente. Estas estrategias variaron en términos de duración enfocándose en tácticas a corto y mediano plazo- y de niveles de institucionalización, objetivos y escalas (Ruibal y Fernandez Anderson, 2018, 2).

Las tácticas de los movimientos sociales incluyeron: primero, abogar por proyectos de ley de aborto desde el año 2007; segundo, alentar la movilización política buscando involucrar a los funcionarios oficiales en miras a que se comprometan a reducir la mortalidad (Organización Mundial de la Salud, 2012, 17 y 90) causada por abortos inseguros (Bergallo, 2014, 247); tercero, la estrategia de provisión de servicios de aborto, para saldar la falta de implementación efectiva en los hospitales públicos (Bergallo, 2014, 247); y cuarto, el inicio de litigios judiciales bajo el Artículo 86 del Código Penal. El resultado de la judicialización fue la consistente aceptación de los jueces en aplicar las circunstancias cubiertas por Código Penal, legitimando el valor de la norma formal y fomentando la redacción de guías para la provisión de abortos legítimos a ser adoptados por los Ministerios de Salud. ${ }^{12}$

La primera y última de las tácticas, también conocidas como "movilización legal", son ejemplos de abordajes que hacen converger las acciones de los movimientos sociales y los reclamos legales (Ruibal, 2015). En América Latina, desde el restablecimiento de las

12 Análisis jurisprudencial profundizado en: Edurne Cárdenas y Leah Tandeter, Derechos sexuales y reproductivos en argentina: una revisión de la legislación y la jurisprudencia (CLADEM Argentina Comité de América Latina y el Caribe para la Defensa de los Derechos de la Mujer, 2008). Disponible en: https://argentina.unfpa.org/sites/default/files/pub-pdf/Conders DSR-Legislacion-yJurisprudencia-en-Argentina.pdf 
democracias, los movimientos sociales desarrollaron nuevas vertientes de incidencia y utilizaron el discurso constitucional y de derechos humanos a los fines de sustentar sus reclamos y acciones colectivas (Ruibal, 20015). En particular, la judicialización y el reclamo por la legalización del aborto han provocado nuevos procesos de movilización legal feminista. Para tener este tipo de movilización, las organizaciones de base resultan actores clave para la promoción y protección de derechos humanos, así como también son esenciales para proveer medios y conocimiento técnico para involucrarse en litigios (Ruibal, 2015).

Asimismo, la táctica de provisión de servicio —no estando estrictamente encuadrada como estrategia legal- merece varias consideraciones. Las mencionadas "redes de rescate" que desarrollaron esta línea son esencialmente ONGs involucradas con la acción directa de provisión de servicios, apuntando a hacer el aborto seguro y accesible a toda mujer que tuviera embarazos no deseados y proveyendo información sobre educación sexual y reproductiva. Las organizaciones no se enfocaron en la reforma legal, sino que buscaron incrementar el acceso en la práctica para combatir la prohibición informal vigente. Resulta destacable que, ignorando la interpretación dominante de la legislación vigente, estos grupos desarrollaron una interpretación autónoma del artículo 86 del Código Penal aferrándose al entendimiento amplio y comprehensivo de la "salud de la mujer" incluyendo su faceta psicológica, entre otros elementos. Esta interpretación se basó en la definición amplia de salud de la Organización Mundial de la Salud (CELS, 2018a) y, en consecuencia, el activismo encontró la forma de reconocer el aborto bajo toda circunstancia, mostrando un discurso basado en la interpretación de un instrumento internacional y reflejando su intención de recurrir a nociones legales para justificarlo. Por lo tanto, no tratándose netamente de una línea de incidencia legal, sí se puede identificar claramente el uso del marco internacional y lenguaje de derechos para escudar una práctica que contravenía la interpretación hegemónica del concepto de salud. 
El trabajo de campo realizado por las organizaciones, desde estos y otros múltiples ángulos, impactó en la remoción del aborto de la total clandestinidad y lo ubicó en un espacio semipúblico (Ruibal y Fernández Peterson, 2018, 10). De esta manera, dichas líneas de activismo produjeron un profundo impacto en términos de legitimación de la práctica, lo cual contribuyó a crear las condiciones para el cambio legislativo.

\section{El "hito" judicial}

Basándose en este camino histórico, legal y social, la sentencia 'F., A. L.' fue un caso clave, decidido a principios del 2012 por la Corte Suprema de Justicia de la Nación (CSJN), que favoreció el clima para una reforma legal. ${ }^{13}$ A grandes rasgos, la sentencia versó sobre el aborto no punible en favor de una joven que fue violada por un familiar a los 15 años y que buscaba interrumpir su embarazo en un hospital público. El fallo no solamente es crucial porque significó un paso hacia el acceso al aborto, sino que también su peso radica en la importante función que tiene la judicialización de los derechos humanos en razón del control de convencionalidad introducido en la reforma constitucional de 1994, tal como fue mencionado anteriormente.

Para comenzar, la CSJN abordó la alegada incompatibilidad entre las causales justificadas de aborto bajo el Artículo 86 del Código Penal y la protección del derecho a la vida bajo la Convención Americana de Derechos Humanos (CADH). La CSJN se basó en el caso de 1981 de la Comisión Interamericana de Derechos Humanos “Baby Boy"14 a los fines de indicar que la redacción de la $\mathrm{CADH}$, en su artículo 4, no implicaba una interpretación unívoca de que el derecho

\footnotetext{
${ }^{13}$ CSJN, 12 de marzo de 2012, F., A.L., Expediente Letra “F,” No. 259, Libro XLVI, (Arg.).

14 Comisión Interamericana de Derechos Humanos, Caso 2141, 'Baby Boy’ (Estados Unidos de América), Res. No. 23/81, OAS/Ser.L/VIII.52, doc. 48 (1981), disponible en: https://www.cidh.oas.org/annualrep/80.81sp/EstadosUnidos2141.htm
} 
a la vida era desde la concepción. Considerando que el artículo 4 usa la terminología “en general” (Pou Giménez, 2019, 116-117), se puede afirmar que éste permite la coexistencia entre la noción de la vida desde la concepción y las regulaciones admitiendo la descriminalización del aborto. ${ }^{15}$

A través de la sentencia 'F., A. L.', la CSJN también aplicó el control de convencionalidad, que ya se venía realizando, pero en esa oportunidad al interpretar la normativa aplicable en materia de aborto. En ese sentido, la CSJN recurrió a instrumentos internacionales como la Convención sobre la Eliminación de todas formas de Discriminación contra la Mujer (CEDAW, por sus siglas en inglés), la Declaración Universal de Derechos Humanos (DUDH), la Convención de los Derechos del Niño (CDN) así como también la Convención Interamericana para Prevenir, Sancionar y Erradicar La Violencia Contra La Mujer (o Convención De Belém Do Pará). Asimismo, la CSJN también refirió a derecho blando o soft law, en particular a decisiones de casos individuales del Comité de Derechos Humanos de las Naciones Unidas, Observaciones Finales y Observaciones Generales. Al hacerlo, la CSJN buscó asegurar el cumplimiento de las obligaciones internacionales de Argentina.

Además, la CSJN emitió una decisión que proveyó una interpretación autoritativa sobre las excepciones provistas en el Código Penal, cuyo entendimiento no se encontraba claro en la ley escrita (Fine, Mayall y Sepúlveda, 2017, 73). La CSJN respaldó la decisión tomada por la Suprema Corte de Chubut (la provincia de donde emergió el caso) y consideró que la autorización para recurrir a un aborto del Artículo 86, inciso 2, no podía ser interpretada como

\footnotetext{
${ }^{15}$ En noviembre de 2012, la Corte Interamericana de Derechos Humanos decidió sobre el caso Artavia Murillo v. Costa Rica, siguiendo el precedente ‘Baby Boy’. Esta decisión disipó definitivamente las dudas respecto a la interpretación del artículo 4 de la CADH. El caso trata de una fertilización in vitro y cubrió la temática del derecho a la vida de los embriones. La Corte interpretó el artículo 4(1) indicando que había una práctica generalizada asociada con el principio gradual e incremental —en vez de absoluto — de protección de la vida prenatal. Disponible en: http://www.corteidh.or.cr/ docs/casos/articulos/seriec 257 ing.pdf
} 
dos requisitos cumulativos - violación y deficiencia mental de la mujer - para acceder a un aborto legal. Esencialmente, la CSJN concluyó que el hecho de haber experimentado una violación sexual bastaba para autorizar un aborto independientemente de las capacidades mentales de la víctima, en línea con el cuerpo de jurisprudencia de las provincias, protocolos y regulaciones nacionales de salud (Fine, Mayall, Sepúlveda, 2017). Decidir de otra manera supondría una carga inconmensurable (Pou Giménez, 2019) y hasta una demanda heroica (Bergallo, 2014, 265) sobre las mujeres, resaltando así la CSJN el valor de la dignidad.

A pesar de que el aspecto sustantivo aparece limitado en la sentencia, ${ }^{16}$ la CSJN refirió a nociones de "igualdad", "dignidad", "privacidad" y "salud” y determinó que la judicialización en el acceso al aborto era innecesaria y una práctica ilegal que negaba derechos básicos (Pou Giménez, 2019). Desde este punto de vista, la CSJN subrayó la importancia de la aplicación de la ley formal y la necesidad de remover los obstáculos institucionales que dificultaban el acceso a los abortos del Artículo 86 (Bergallo, 2014, 266) del Código Penal.

La CSJN, sin embargo, no sustentó su decisión utilizando un abordaje de autodeterminación individual, reproductiva y ejercicio de la autonomía, que podría haber sido usado en aplicación del artículo 19 de la Constitución Argentina, por ejemplo. Este artículo, cuando fue sucintamente mencionado, ${ }^{17}$ fue interpretado en su versión limitada de "principio de reserva", esencialmente, refiriéndose al derecho a la privacidad.

En contraste, puede ser valorado positivamente que la CSJN haya indicado que el Estado debía “poner a disposición, de quien solicita la práctica, las condiciones médicas e higiénicas necesarias para llevarlo

\footnotetext{
${ }^{16}$ Enfoque conocido como "minimalismo judicial".

${ }^{17}$ CSJN, 12 de marzo de 2012, F., A.L., Expediente Letra “F,” No. 259, Libro XLVI, (Arg.), cons. 20.
} 
a cabo de manera rápida, accesible y segura"18 sin cargar desproporcionadamente a las mujeres. La CSJN también llamó a las distintas instituciones involucradas a facilitar el acceso e identificar acciones concretas para lograrlo (Pou Giménez, 2019).

La CSJN acogió el enfoque de la "proceduralización" (Bergallo, $2014,268)$ que ya había emergido desde el 2000, hacia el restablecimiento de la ley formal (el ignorado artículo 86 del Código Penal). No obstante, este abordaje procedimental naturalmente presenta ciertas fragilidades en términos de jerarquía legal, riesgos en la implementación debido al carácter federal del país, entre otras dificultades. Por lo tanto, la lucha por el aborto legal prosiguió y presentar un proyecto de Ley nacional se tornó en el nuevo norte para los años siguientes, hasta la actualidad, de activistas, defensores y defensoras de derechos humanos y de derechos de las mujeres.

\section{b. 2018: El proyecto de ley de aborto}

En el año 2018, por primera vez, el proyecto de ley de aborto fue debatido ante el Congreso luego de más de una década ${ }^{19}$ de ser propuesto por "La Campaña". ${ }^{20} \mathrm{El}$ tratamiento de la ley también fue acompañado por un movimiento popular sin precedentes, que comenzó alrededor del año 2003 por agrupaciones como "La Campaña", "Encuentro Nacional de Mujeres"21 y "Ni una menos", ${ }^{22}$ que surgió posteriormente. Los movimientos de mujeres no

\footnotetext{
${ }^{18}$ CSJN, 12 de marzo de 2012, F., A.L., Expediente Letra “F," No. 259, Libro XLVI, (Arg.), cons. 25.

${ }^{19}$ Fue presentado por primera vez el 28 de mayo de 2007. Campaña Nacional por el Derecho al Aborto Legal, Seguro y Gratuito. Más información disponible en:

http://www.abortolegal.com.ar/proyecto-de-ley-de-legalizaciondespenalizacion-delaborto-en-argentina/

${ }^{20}$ Campaña Nacional por el Derecho al Aborto Legal, Seguro y Gratuito, sitio oficial. Más información disponible en: http://www.abortolegal.com.ar/about/

${ }^{21}$ Encuentro Nacional de Mujeres, sitio oficial: http://encuentrodemujeres.com.ar/

$22 \mathrm{Ni}$ Una Menos, sitio oficial: http://niunamenos.org.ar/quienes-somos/cartaorganica/
} 
solamente apoyaban la legalización del aborto en particular, sino también los derechos de las mujeres, los derechos del colectivo LGBTIQ+y la diversidad sexual, en general.

En relación al proyecto de ley en concreto, este ofrecía cambiar de un modelo de descriminalización parcial basado en razones estrictas a un modelo combinado de interrupción voluntaria del embarazo (IVE) que permitía el aborto bajo solicitud durante las primeras 14 semanas (la catorceava inclusive) y, luego de ese período de tiempo, el aborto solo se podía solicitar por la mujer (o cuerpo gestante) bajo casos específicos, como violación, riesgo en la salud o vida o inviabilidad del feto en la vida extrauterina (Honorable Senado de la Nación, 2018).

Tal como indican los fundamentos acercados por "La Campaña”, el objetivo de la reforma legal es el de "reconocer a las mujeres como sujetos sociales, como ciudadanas y agentes morales capaces de decidir, si desean o no ser madres, el número de hijos y el espacio entre los nacimientos." ${ }^{23}$ Asimismo, se aclara que el artículo primero del proyecto busca delimitar legalmente un período de tiempo de 14 semanas, "En concordancia con el marco jurídico de la mayoría de los países que tienen aborto legal (...) salvo en casos de violación, peligro para la salud o vida de la persona gestante, o malformaciones fetales graves, en que este límite queda sin efecto."24

\section{c. El debate}

Desde abril hasta agosto de 2018, el Congreso Nacional argentino tuvo la oportunidad de participar en un debate abierto sobre el aborto que incluyó a más de 700 expositores y expositoras para la Cámara de

${ }^{23}$ Campaña por el derecho al aborto legal, seguro y gratuito - Fundamentos Proyecto de Ley (2011). Disponible en: http://www.abortolegal.com.ar/wpcontent/uploads/2011/08/Fundamentos-y-Proyecto-Ley-IVE.pdf

${ }^{24}$ Campaña por el derecho al aborto legal, seguro y gratuito. Hacia la Legalización de la Interrupción Voluntaria del Embarazo en la Argentina. Argumentos para el debate (2018). http://www.abortolegal.com.ar/wp-content/uploads/2018/04/ArgumentosCampan\%CC\%83a-2018-FFF.pdf 
Diputados y más de 60 para la Cámara de Senadores. Miembros del Congreso del mismo partido político se dividieron entre aquellos que apoyaban y aquellos que se oponían al proyecto de ley. Como contracara, surgieron alianzas interpartidarias debido a la afinidad en las visiones de distintos miembros del parlamento que pertenecían a corrientes políticas diferentes.

Mientras tanto, organizaciones relevantes de la sociedad civil ${ }^{25}$ monitorearon las exposiciones y diseminaron la información a través de informes (ELA, REDAAS, CEDES, 2018) en los que rastrearon y sistematizaron los argumentos principales que fueron avanzados por expositores y expositoras. Con relación a los argumentos legales, tanto las personas expertas que apoyaban como aquellas que cuestionaban el proyecto de ley, basaban sus argumentos primordialmente en la Constitución Nacional y en los tratados internacionales de derechos humanos que Argentina ratificó.

Para aquellos oponiéndose al proyecto de ley de aborto, sus justificaciones incluían la protección de la vida desde la concepción en línea con la $\mathrm{CADH}$; críticas al mencionado fallo 'F., A. L.'; la imposición del DIDH del deber de proteger a la parte más débil, en este caso el no nacido; la falta de obligación de legalizar el aborto por parte del DIDH y la falta de reconocimiento del derecho al aborto legal a nivel internacional (ELA, REDAAS, CEDES, 2018), entre otros argumentos.

Por otra parte, prominentes juristas posicionándose a favor del proyecto de ley, ${ }^{26}$ indicaron las razones por las cuales el acceso al aborto legal no contravenía los estándares internacionales de derechos humanos y que el DIDH no se presentaba como un

\footnotetext{
${ }^{25}$ Las organizaciones involucradas eran ELA, REDAAS y CEDES (las siglas de las organizaciones ya mencionadas).

${ }^{26}$ Como Mónica Pinto, profesora de DIDH y exdecana de la Facultad de Derecho de la Universidad de Buenos Aires. Exposición completa disponible en: https://www.youtube.com/watch?v=mW6xnSPE2g0; y Fabián Salvioli, profesor de DIDH y exmiembro del Comité de las Naciones Unidas de Derechos Humanos. Exposición completa disponible en: https://www.youtube.com/watch?v=MlfBhle3tws
} 
obstáculo para la adopción del proyecto de ley de IVE. Indicaron también que el Comité de Derechos Humanos, el Comité DESC y el Comité CEDAW, felicitaron y saludaron a los Estados parte que establecieron leyes de IVE, como fuera el caso de Uruguay.

Esto parece demostrar que los instrumentos formales de derechos humanos fueron usados como una suerte de "comodín" para justificar posiciones antagónicas, mientras que trabajos más precisos de los órganos de tratados de derechos humanos fueron presentados únicamente como formas de justificar el avance del proyecto de IVE.

Tras el amplio debate y las exposiciones indicadas, la Cámara de Diputados aprobó el proyecto de ley por un margen ajustado de 129 votos a favor y 125 votos en contra, el 14 de junio de 2018 y la Cámara de Senadores rechazó el proyecto de IVE, también por una diferencia ajustada, de 38 votos en contra y 31 votos a favor, el 8 de agosto de 2018.

Pese a la aprobación ajustada en la Cámara de Diputados, es dable remarcar la presión de grupos conservadores en la esfera civil, con amplias redes en la esfera política —especialmente a nivel provincial- contribuyeron a que la Cámara Alta finalmente rechazara el proyecto por una diferencia de tan solo siete votos. Esto sirve para resaltar que mientras que los movimientos proaborto han abogado por el avance de la reforma legal, tal como fue descrito, otros actores de la sociedad civil, que se oponían al proyecto de ley, también interactuaron a nivel social, civil y político e incidieron para contrarrestar las iniciativas de reforma legislativa.

\section{d. Últimos avances y consideraciones finales}

El pasado 28 de mayo de 2019 el proyecto de ley de IVE fue presentado nuevamente ante el Congreso Nacional. Puesto que el país atravesó un año electoral en 2019, los y las votantes — sobre todo mujeres y jóvenes - reclamaron que los candidatos presidenciales se 
posicionen con relación a esta temática, en miras a nuevo debate ante las Cámaras en el 2020.

Una alternativa posible que se está discutiendo es la descriminalización del aborto, pero sin ley de IVE. Se trata de una posición intermedia que el electo presidente indicó durante su campaña (El País, 2019) que pretendería adoptar.

A pesar de cuán decepcionante se muestra la opción de la mera descriminalización en el marco de la lucha por el aborto legal, esto aún representa un paso hacia adelante considerando la situación precaria que prevalece actualmente. También se encuentra la opción de que se vuelva a debatir el proyecto de ley de aborto (con las modificaciones introducidas) y se vote nuevamente con la nueva composición de congresistas tras las recientemente concluidas elecciones.

Asimismo, ha habido vaivenes en noviembre de 2019 en relación con el avance por la —en ese entonces- Secretaría de Salud de un protocolo actualizado de interrupción legal del embarazo, que buscaba incorporar elementos que ampliarían el acceso al aborto (Clarín, 2019b). Tras la derogación de esta iniciativa, el Secretario de Salud renunció a su cargo a fines de noviembre, poco antes del fin del mandato del gobierno de turno (Clarín, 2019c).

A pocos días de asumir el nuevo presidente, a mediados de diciembre de 2019, se aprobó un protocolo que flexibiliza el aborto no punible bajo las causales ya previstas por la ley (Clarín, 2019d y El Cronista, 2019), inspirado en el protocolo recientemente derogado.

El avance de este protocolo implica un progreso en pos de la liberalización y poseerá claros efectos prácticos, tangibles y beneficiosos que favorecerá el acceso. Sin embargo, este paso sigue en la línea del abordaje procedimental y, por lo tanto, adolece de ciertos defectos: débil jerarquía legal, riesgos en la implementación a nivel nacional debido al sistema federal que puede conducir a fragmentación y menos seguridad jurídica, entre otros. 


\section{El contraste de la experiencia nacional a la luz de los estándares internacionales}

Relevante a este artículo, una pregunta permanece sin ser contestada: si el DIDH fue usado como Caballo de Troya por los parlamentarios en el caso argentino, más allá de sus posiciones de apoyo u oposición al proyecto de ley, iHasta qué punto las campañas nacionales y de incidencia por el derecho al aborto legal deberían utilizar el marco normativo del DIDH para definir y sustentar su defensa y promoción de mejores leyes nacionales de aborto?

\section{a. Panorama general}

La evolución del DIDH ha presentado una relación compleja con los derechos de las mujeres en general, y con el aborto en particular (Sedacca, 2017, 111). El DIDH ha recibido críticas feministas que afirman que los derechos humanos se encuentran predominantemente orientados a los hombres desde su concepción y en su implementación (Dembour, 2018, 53). Adicionalmente, estas críticas también han resaltado que la clásica división del DIDH entre la esfera pública y privada ha perjudicado históricamente a las mujeres.

Un aspecto relevante por considerar es que, en estadios posteriores, el DIDH fue desarrollando una tendencia a cargar a las autoridades públicas con responsabilidades (acciones afirmativas) que aparentemente solo pueden ser asumidas por el Estado (Tomuschat, 2008, 5). El Comité CEDAW es un claro ejemplo, pues requiere que todos los Estados parte de la CEDAW modifiquen los patrones sociales y culturales que estereotipan y crean roles de género, ${ }^{27}$ adoptando un enfoque vertical descendente (Tomuschat, 2008) - denominado top-down en inglés- que parte del plano

${ }^{27}$ Convención sobre la Eliminación de todas formas de Discriminación contra la Mujer, artículo 5 (a). 
supranacional y busca imponer obligaciones a los Estados, que luego recaerán sobre la sociedad. La idoneidad de este enfoque será problematizada en las próximas líneas.

En lo relativo al aborto, tal como identificó la doctrina "el alcance con el que el derecho internacional debe proteger al no nacido, y por lo tanto permitir o prohibir el aborto, estuvo sujeto a un significativo debate durante la redacción de las provisiones sobre el derecho en varios instrumentos internacionales" (Mitra, 2017, 145). Esto ocurrió en la redacción de la DUDH, del Pacto Internacional de Derechos Civiles y Políticos (PIDCP), de la Declaración de los Derechos del Niño y la Convención de los Derechos del Niño, para dar algunos ejemplos (Mitra, 2017, 148-9).

En la negociación de estos instrumentos, llegar a un acuerdo respecto al momento desde el cual comienza la vida y ésta debe ser protegida, resultó una cuestión sumamente compleja. En esa época, algunos países ya habían permitido el aborto bajo distintos tipos de circunstancias (incluso Argentina, a pesar de la falta de acceso en la práctica) mientras que había otros que lo criminalizaban de manera absoluta. Por lo tanto, el resultado de estas negociaciones fue que los instrumentos permanecieran lo suficientemente generales y abiertos para ser interpretados de manera consistente con las leyes domésticas (Mitra, 2017, 146) de los Estados y así lograr consenso y una amplia ratificación. De esta manera, la redacción de los instrumentos fundacionales del DIDH revela una práctica en la que opta por no zanjar la cuestión a nivel internacional y, en cambio, relega las cuestiones relacionadas con el aborto a la esfera de las legislaciones domésticas (Mitra, 2017, 145) de cada Estado en particular.

Esta ambigüedad del DIDH respecto al aborto como una práctica y como un derecho, produjo grandes vacíos legales que luego serían colmados por los mecanismos convencionales - en esencia, los Comités de tratados de Naciones Unidas. Estos Comités han desarrollado interpretaciones respecto a la terminación del embarazo y el aborto cuando fue necesario, por ejemplo, al decidir en 
Comunicaciones Individuales y al realizar revisiones periódicas de los Estados parte de las Convenciones (a través de Observaciones Finales). En general, el trabajo de los Comités se inclinó por promover el acceso al aborto en ciertas circunstancias.

Adicionalmente, las agencias especializadas de Naciones Unidas, como la Organización Mundial para la Salud (OMS) y expertos independientes de Naciones Unidas, también contribuyeron en proveer guías y lineamientos relacionados con este tema. Algunos de los instrumentos más importantes que hayan referido al aborto de distintas maneras podrán ser encontrados a continuación.

En la Observación General Nro. 22 sobre derechos sexuales y reproductivos del Comité de Derechos Económicos, Sociales y Culturales (DESC), se refirió extensivamente al aborto y a los aspectos relacionados con los derechos civiles y políticos, reconociendo que ambos conjuntos de derechos están relacionados y aún más en esta materia. El Comité DESC también expresó que los Estados deben combatir todos los estereotipos discriminatorios (Comité DESC Observación General 22, 2016, pp. 33 y 36), siguiendo el ya aludido enfoque vertical descendente de derechos humanos.

En el marco de la CEDAW, la Recomendación General Nro. 30 sobre las mujeres en la prevención de conflictos y en situaciones de conflicto y posteriores a conflictos, en donde el Comité CEDAW recomendó, en varias secciones, que los Estados partes provean acceso a servicios de aborto seguro y prevengan embarazos forzosos (Comité CEDAW Recomendación General 30, 2013).

Otro ejemplo es el Comité de los Derechos del Niño que en su Observación General Nro. 4, sección 4, dedicada a la información, desarrollo de aptitudes, asesoramiento y servicios de salud, indicó que: "Los Estados Partes deben adoptar medidas para reducir la morbimortalidad materna y la mortalidad de las niñas adolescentes, producida especialmente por el embarazo y las prácticas de aborto peligrosas, y prestar apoyo a los padres de las adolescentes." (Comité CDN Observación General 4, 2003, pp. 31). 
A pesar de ciertamente no haber agotado los instrumentos emitidos por los Comités de derechos humanos relevantes a la temática y solo haber mencionado algunos de los instrumentos más recientes, el trabajo de otros mecanismos extra-convencionales de Naciones Unidas deberían ser abordados dado que reforzaron la labor de los Comités.

Un ejemplo es el informe del Relator Especial sobre la tortura y otros tratos o penas crueles, inhumanos o degradantes. Este experto concluyó que los Estados debían realizar acciones afirmativas tendientes a reformar las legislaciones restrictivas relativas al aborto y garantizar el tratamiento inmediato e incondicional a personas precisando cuidados médicos de emergencia, incluso siendo resultado de abortos ilegales (Informe Relator Tortura, 2016).

De manera similar, el Relator Especial sobre el derecho de toda persona al disfrute del más alto nivel posible de salud física y mental de las Naciones Unidas, indicó que la denegación en el acceso al aborto perjudica los derechos de las niñas y adolescentes y enfatizó la importancia de descriminalizar el aborto (Informe Relator Salud, 2016).

En la misma línea, la agencia especializada de Naciones Unidas en materia de salud, la OMS, elaboró la ya mencionada definición comprehensiva de salud (OMS Constitución, 1946). Asimismo, la organización emitió numerosas guías, entre las cuales están incluidas las recomendaciones para el manejo médico del aborto (OMS Tratamiento médico del aborto, 2018).

Una vez más refiriéndose a mecanismos extra-convencionales, el Consejo de Derechos Humanos a través del proceso de examen periódico universal sobre todos los Estados miembros de las Naciones Unidas y a través de sus resoluciones, utiliza estas instancias para generar recomendaciones relativas a esta temática ${ }^{28}$.

${ }^{28}$ A modo ilustrativo, ver la nota siguiente en la que los grupos de trabajo del Consejo de Derechos Humanos han estado involucrados. International Safe Abortion Day- 
Por último, y en este caso retornando a los mecanismos convencionales, numerosas Observaciones Finales recientes refieren a la terminación voluntaria del embarazo al realizar el examen de los Estados, principal pero no solamente, recomendando la descriminalización del aborto y su acceso bajo ciertas circunstancias ${ }^{29}$ y expresando preocupación por las legislaciones restrictivas cuyo resultado es el incremento de abortos inseguros y el potencial encarcelamiento de las mujeres, aún en casos de abortos espontáneos (CDH, Observaciones Finales Salvador, 2018, pp. 15).

A la luz de esto, se hace evidente la importancia del trabajo efectuado al momento por los mecanismos internacionales, los procedimientos y las agencias especializadas. Han promovido la interpretación de que, bajo ciertas circunstancias, la negativa a llevar a cabo un aborto conlleva la violación de derechos. En la práctica de estos órganos, el aborto no aparece como un derecho independiente; en su lugar, cuando se deciden casos de acceso al aborto, su defensa se encuentra mayormente justificada en la conexión con otros derechos (Joyce, 2017) como no ser sometida a malos tratos, el derecho a la salud, el derecho a la vida, entre otros. Sin embargo, hay un reconocimiento implícito del derecho al aborto en ciertas circunstancias.

\section{b. Caso de estudio de los órganos de tratados: El Comité de Derechos Humanos}

La Observación General Nro. 36 (OG36), sobre el derecho a la vida, es el instrumento más reciente adoptado por el Comité de Derechos Humanos (CDH) y que hace directa referencia al aborto.

Friday 28 September 2018, disponible en: https://www.ohchr.org/EN/NewsEvents/ Pages/Display News.aspx?NewsID=23644\&LangID=E

${ }^{29}$ Por ejemplo, en 2018 el Comité de Derechos Humanos refirió a la terminación voluntaria del embarazo en las Observaciones Finales de 10 de un total de 16 países bajo examen: Belice, Guinea, República Democrática Popular Lao, Sudán, Gambia, Liberia, Argelia, El Salvador, Líbano y Guatemala. 
La OG Nro. 36 reemplaza las previas Observaciones Generales Nro. 6 y Nro. 14, emitidas por el mismo órgano. En oposición al campo de aplicación limitado de las Observaciones Generales anteriores, la OG36 es una herramienta legal comprehensiva y abarca una amplia gama de interpretaciones y consideraciones respecto al derecho a la vida. El instrumento se encuentra alineado con el desarrollo progresivo de los derechos humanos, su expansión y su creciente complejidad al definirlos. Asimismo, el enfoque de la OG36 se encuentra basado en la premisa del derecho a la vida en dignidad..$^{30}$

En el extenso párrafo $\mathrm{N}^{\circ} 8$ de la versión en inglés, ${ }^{31}$ el CDH abordó la terminación voluntaria del embarazo. Habiendo sido uno de los

\footnotetext{
${ }^{30}$ Tal como surge de la nota "El comentario general envió un mensaje fuerte contra la interpretación reducida del derecho a la vida, tal como es apropiado en un mundo globalizado, y subrayó el derecho a la vida con dignidad." La versión original reza: “The General Comment sent a strong message against the narrow legal interpretation of the right to life, as was appropriate in a globalized world, and it underscored the right to life with dignity". Disponible en: https://www.ohchr.org/EN/NewsEvents/Pages/ DisplayNews.aspx?NewsID=23797\&LangID=E

${ }^{31}$ Se trata del párrafo número 9 en la versión en español, la cual no es la traducción de la última versión oficial de la OG36, que se encuentra disponible solamente en inglés (a enero de 2020). A continuación, su transcripción en el idioma oficial:
}

"8. Although States parties may adopt measures designed to regulate voluntary terminations of pregnancy, such measures must not result in violation of the right to life of a pregnant woman or girl, or her other rights under the Covenant. Thus, restrictions on the ability of women or girls to seek abortion must not, inter alia, jeopardize their lives, subject them to physical or mental pain or suffering which violates article 7 , discriminate against them or arbitrarily interfere with their privacy. States parties must provide safe, legal and effective access to abortion where the life and health of the pregnant woman or girl is at risk, or where carrying a pregnancy to term would cause the pregnant woman or girl substantial pain or suffering, most notably where the pregnancy is the result of rape or incest or is not viable. [8] In addition, States parties may not regulate pregnancy or abortion in all other cases in a manner that runs contrary to their duty to ensure that women and girls do not have to undertake unsafe abortions, and they should revise their abortion laws accordingly. [9] For example, they should not take measures such as criminalizing pregnancies by unmarried women or apply criminal sanctions against women and girls undergoing abortion [10] or against medical service providers assisting them in doing so, since taking such measures compel women and girls to resort to unsafe abortion. States parties should not introduce new barriers and should remove existing barriers [11] that deny effective access by women and girls to safe and legal abortion [12], including barriers caused as a result of the exercise of conscientious objection by individual medical providers. [13] 
segmentos más controversiales y debatidos, el CDH adoptó una posición en favor de los derechos de las mujeres. A pesar de que el aborto no es concebido como un derecho en sí mismo, la observación general fomenta el acceso a ciertas prácticas de terminación voluntaria del embarazo en mayor o menor grado. El CDH refiere a ciertas medidas que los Estados partes deben tomar usando palabras como "puede" o "debe" según la recomendación que se trate, reflejando una redacción cuidadosa ${ }^{32}$ y un nivel de obligación diferenciado.

Una de las recomendaciones es que "los Estados partes no deben introducir barreras y deben remover las barreras existentes que deniegan el acceso efectivo de las mujeres y niñas al aborto legal" ${ }^{33} \mathrm{~A}$ pesar de que el término "aborto legal" se encuentra presente, siempre se halla limitado y matizado por las circunstancias específicas.

Asimismo, el CDH indicó que "los Estados partes no deben regular el embarazo ni el aborto de manera contraria a su deber de velar por que las mujeres no tengan que recurrir a abortos peligrosos, y deben revisar sus leyes de aborto concordantemente". ${ }^{34}$ Esta recomendación reconoce la cantidad masiva de abortos clandestinos

States parties should also effectively protect the lives of women and girls against the mental and physical health risks associated with unsafe abortions. In particular, they should ensure access for women and men, and, especially, girls and boys, [14] to quality and evidence-based information and education about sexual and reproductive health [15] and to a wide range of affordable contraceptive methods, [16] and prevent the stigmatization of women and girls seeking abortion. [17] States parties should ensure the availability of, and effective access to, quality prenatal and post-abortion health care for women and girls, [18] in all circumstances, and on a confidential basis. [19]"

$32 \mathrm{El}$ análisis de la redacción está basado en una traducción no formal del párrafo 8, al no haber una traducción oficial de la versión final de la Observación General 36 al español.

33 Ibid. La versión original dice: "States parties should not introduce barriers and should remove existing barriers that deny effective access by women and girls to safe and legal abortion".

${ }^{34}$ Ibid. La versión original estipula: "States parties may not regulate pregnancy or abortion in all other cases in a manner that runs contrary to their duty to ensure that women and girls do not have to undertake unsafe abortions, and they should revise their abortion laws accordingly". 
como una importante razón de mortalidad de las mujeres e invita a la reforma legal, sin embargo, su fuerza obligatoria es débil. Es notable que esta recomendación, en particular, se encuentra basada en las Observaciones Finales de varios países ante el Comité, incluyendo el caso del examen de Argentina de 2010.

Por último, la OG36 establece que el aborto no debe ser criminalizado o sancionado, incluyendo a las mujeres que recurren a la práctica como al personal médicos que las asisten. ${ }^{35}$ Esto no es un aspecto novedoso a nivel internacional, pero su introducción en la OG continúa y reafirma la tendencia.

Respecto al impacto de la OG36, actores tales como relevantes ONGs de derechos sexuales y reproductivos ${ }^{36}$ también proveyeron su interpretación y consideraron que la redacción de la OG36 indica que el aborto es un derecho humano. De acuerdo con su visión, el CDH promueve el establecimiento y acceso al aborto legal y se espera que los movimientos sociales y populares, las ONGs internacionales, regionales $\mathrm{y}$ locales aprovechen el instrumento como una herramienta que provee un sólido apoyo para "el cabildeo, el litigio, la investigación que define y fortalece el aborto como un derecho fundamental". ${ }^{37}$ En contraposición, nada impide que grupos en oposición a esta reforma puedan usar la misma redacción del párrafo 8 de la OG36 para indicar que no se ha establecido un derecho explícito al aborto legal en toda circunstancia que esté por fuera de las que fueron específicamente indicadas en la OG36.

\footnotetext{
35 Ibid.

36 Por ejemplo, el “Centro por los Derechos Reproductivos” (CDR), la organización que defendió a la señora Mellet en el conocido caso contra Irlanda ante el CDH.

37 Traducción de la autora. Comentario original: “...advocacy, litigation, and research that defines and enforces abortion as a fundamental human right". Sitio oficial del Centro de Derechos Reproductivos, "UN Human Rights Committee Asserts that Access to Abortion and Prevention of Maternal Mortality are Human Rights", 31 de Octubre de 2018, disponible en:

https://reproductiverights.org/press-room/un-human-rights-committee-assertsaccess-abortion-and-prevention-maternal-mortality-are
} 
Una vez más, a pesar de que la OG36 es una observación general muy reciente y provee un nivel de detalle mayor en esta temática, un alto grado de margen permanece en manos de los Estados debido a la falta de consenso en la temática. En pocas palabras, no se proclamó o reconoció un derecho autónomo al aborto legal a nivel internacional, pero se indicó que muchas circunstancias pueden conducir a que se conceda acceso al aborto.

El hecho de que la OG36 aporte este enfoque matizado, donde la perspectiva de derechos de la mujer se encuentra presente pero aun así se ve coartada en el acceso al aborto salvo circunstancias específicas, posee tanto aspectos positivos como negativos. Por un lado, un logro es que esta posición se presenta como una base que es más proclive a generar consenso ante posiciones antagónicas; por el otro, este abordaje no provee un piso firme para realizar incidencia por leyes de acceso irrestricto al aborto durante un período de tiempo determinado (por ejemplo, las 14 semanas, en línea con el caso de proyecto de ley de Argentina) como a las que apuntaría el activismo proaborto.

De esta manera, la herramienta provee cimientos poderosos en contextos normativos extremos, tales como legislaciones de prohibición total de acceso al aborto porque no respetar determinados estándares puede conducir a la violación de derechos fundamentales. Sin embargo, pierde su potencial para los casos en que los movimientos sociales aspiren a normativas de empoderamiento de la mujer y de libertad reproductiva.

\section{c. Conclusión de la sección}

Este recorrido, descrito escuetamente, muestra que el DIDH inicialmente fue ambiguo y optó por no tomar una posición respecto al aborto, a los fines de lograr un consenso mayor, y se inclinó por diferir esta cuestión a los Estados. A través del tiempo, el DIDH — por medio de sus mecanismos convencionales y extraconvencionales- 
comenzó a vincular la denegación en el acceso al aborto con distintas violaciones de derechos humanos.

Adicionalmente, el hecho de que las Observaciones Generales, Observaciones Finales y Comunicaciones Individuales emitidas por el $\mathrm{CDH} y$ otros organismos de monitoreo son formalmente no vinculantes (Kälin y Künzli, 2009, 37), se debe tener también en consideración. Por un lado, los instrumentos de soft law-debido a su valor persuasivo- se suelen usar usualmente como una manera de desarrollar el derecho y, asimismo, actualmente este tipo de instancia resulta la más exitosa en invitar a los Estados y actores internacionales a cooperar e involucrarse en negociaciones de instrumentos internacionales. Por otro lado, esta manera de dar forma al derecho se encuentra históricamente caracterizada por su débil obligatoriedad legal y su bajo nivel de compromiso, sobre todo si un Estado no está en línea con lo que aquellos instrumentos prescriben.

Estos elementos — vaguedad, débil obligatoriedad, entre otrosaportan al análisis sobre las razones por las cuales los partidarios del proyecto de ley en Argentina no tenían fundamentos acabadamente sólidos al referirse al ordenamiento del DIDH para defender su posición respecto a la legalización del aborto (que sí tenían para el caso de la descriminalización, por ejemplo). Como resultado, estos determinados aspectos que dan cuenta de la debilidad del DIDH impactaron en el debate nacional.

Teniendo en cuenta estas consideraciones, un punto clave a discutir es hasta qué punto las campañas nacionales por el aborto legal deberían adoptar la normativa vigente de DIDH cuando buscan definir y apoyar estrategias de cabildeo para que haya leyes de aborto a nivel nacional que contemplen mejor sus pretensiones.

\section{Reflexiones finales}

Este artículo mostró la experiencia localizada de la Argentina respecto a la legalización del aborto e ilustró el camino hacia el 
proyecto de ley, que no se aprobó y que fue recientemente reintroducido ante el Congreso Nacional. Las razones del fracaso en la aprobación de la ley son variadas, pero este estudio se enfocó en encontrar la respuesta a la luz del sistema del DIDH.

Para este propósito, en la sección subsiguiente la propuesta fue alejarse (o hacer un zoom out) de la experiencia localizada y presentar lo que estaba ocurriendo a nivel internacional, analizando el progreso y las limitaciones del DIDH. Tal como fue posible entrever, los instrumentos internacionales vinculantes permanecieron silenciosos respecto al aborto y el trabajo realizado por los mecanismos de derechos humanos convencionales y extraconvencionales contribuyó a facilitar el acceso al aborto bajo ciertas circunstancias, a través de sus instrumentos interpretativos. El artículo ha apuntado a mostrar que el DIDH fue (y es) en efecto utilizado como una herramienta de incidencia y campaña; justamente, el DIDH evolucionó de la manera descripta, en parte, debido a su uso como instrumento de cabildeo, que permitió cierta apertura con relación a las circunstancias en que el aborto debe ser accesible a los fines de evitar la violación derechos humanos.

No obstante, el peso de su contribución va variando según a qué tipo de ley se esté apuntando. Si se trata de una ley que pretende incluir mínimos niveles de liberalización, el DIDH es una herramienta ciertamente útil. ${ }^{38}$ En contraste, el caso argentino muestra que el derecho internacional presenta restricciones en cuanto al proyecto de aborto legal e irrestricto en los primeros meses de gestación y aún más en un contexto regional donde hay altos niveles de restricción en esta temática. Ignorar las limitaciones del DIDH debería ser desalentado, puesto que, de tal manera, los movimientos fallarían en hacer frente a dichas restricciones y en tornar al DIDH como una herramienta de cambio que también alcance los derechos de las mujeres, en términos de libertad reproductiva.

38 Tal es así para el caso de países como El Salvador, Nicaragua y Honduras, que presentan este tipo de legislación. 
Derivado de esto, las contribuciones del DIDH en concreto son que: el aborto no debe ser criminalizado, debe ser accesible en ciertas circunstancias y que, como contracara, se considera inaceptable la prohibición total en el acceso. Adicionalmente, la evolución del DIDH llevó a interpretaciones que muestran preocupación por los abortos inseguros y clandestinos, y la desigualdad que eso conlleva, apuntando a evitar el sufrimiento de la mujer embarazada. Como resultado, el DIDH está efectivamente reduciendo el ámbito de libertad de los Estados en sus regulaciones referidas al aborto y a la IVE.

Sin embargo, el análisis sobre el plano internacional reflejó que las circunstancias para que haya aborto legal son limitadas: mayormente vinculadas con derechos específicos tales como la salud, el trato inhumano y degradante y la vida de la mujer, que se verían violados en casos extremos. En otros términos, una cuestión sanitaria que debía ser abordada. Mientras que tales casos son más proclives a generar compasión, estos inevitablemente limitan el éxito en cuestionar las restricciones en el acceso al aborto en un sentido más general (Sedacca, 2017, 117).

Como consecuencia, este enfoque corre el riesgo de dar lugar al imperialismo moral y a descuidar las necesidades de las mujeres quienes, por ejemplo, habiendo tenido sexo consensuado, también tienen razones económicas o un conflicto con sus parejas (Sedacca, 2017) — solo por nombrar algunas de ellas- para terminar sus embarazos. Más aún, esta perspectiva puede perpetuar la idea de que los abortos son aceptables si emanan de buenas razones y que son inaceptables cuando emanan de malas razones (Sedacca, 2017). Asimismo, esta postura también corre el riesgo de descartar la posibilidad de realizar un aborto sin evaluación externa del todo, de parte de los proveedores de la salud (Skuster, 2017) o de la sociedad en general, que ponga en tela de juicio el criterio de la mujer que busca interrumpir su embarazo no deseado. 
Como resultado, una clara debilidad del DIDH es que, debido a esta lógica que subyace, este ordenamiento no provee fundamentos sólidos para pasar de una perspectiva de protección de la salud de la mujer al derecho que ésta tiene de ejercer su libre decisión. En otros términos, el derecho internacional no optó por un enfoque de empoderamiento y ampliación de los espacios de autonomía reproductiva de las mujeres.

En cuanto a si el DIDH podría o debería servir como tal fuerza de empuje para un proyecto de ley nacional como el debatido en Argentina, se puede concluir que un avance más abarcativo (una ley de IVE con causales irrestrictas) todavía enfrenta grandes obstáculos y no se ha alcanzado el grado de madurez del DIDH para que esto suceda. Aún más, tal vez la madurez del DIDH no conduzca inevitablemente a un derecho humano a nivel internacional al aborto legal desde una perspectiva de autonomía reproductiva, porque hacer esto podría implicar inmiscuirse en exceso en cuestiones internas sensibles de los Estados. ${ }^{39}$ En efecto, esto hasta podría ser desalentado porque podría producir un riesgo de deslegitimación, una vez más, del proyecto de los derechos humanos como un todo. ${ }^{40}$

En el estado actual de la situación, el DIDH se encuentra situado en una posición compleja, semejante a la que se enfrentaba en sus inicios: mayor consenso en detrimento de más niveles de defensa de derechos y empoderamiento. La mayor diferencia en la actualidad es que el DIDH pasó en esta materia del silencio a pronunciarse a su favor para determinadas circunstancias, un paso que no debe ser desdeñado. Este avance ya permite contar con herramientas de incidencia y cabildeo para las vertientes proaborto.

En vista de lo anterior, es dable destacar la importancia de los movimientos sociales y de mujeres — desde enfoques de cambio cultural y perspectivas de género de avanzada-como los actores más

\footnotetext{
${ }^{39}$ En línea con el entendimiento del Artículo 2.7. de la Carta de Naciones Unidas.

${ }^{40}$ Para más información sobre la sobreexpansión del DIDH: Hust Hannum, Rescuing Human Rights (Cambridge University Press, 2019).
} 
capacitados para lograr reformas legales hacia el aborto sin invocar causales en el primer trimestre de embarazo, lo cual puede servir como una contribución desde abajo hacia arriba (bottom-up) para los sistemas regionales e internacionales en promover un camino hacia el aborto legal basado en la libertad y autonomía reproductiva de la mujer. Esto refleja que los cambios vinculados a la temática usualmente suceden a nivel microscópico y que este enfoque resulta el predilecto dado que las reformas fluyen desde lo micro hacia lo macro.

Si el DIDH no puede ser el precursor de la legalización total del aborto en los primeros meses de gestación, las sociedades deben generar tal cambio y el DIDH debe acompañarlo, una vez producido. A largo plazo, si el DIDH recibe contribuciones nacionales con una tendencia hacia el aborto legal, se espera que el DIDH sea capaz de lidiar con tal inclinación y pueda contribuir con el empoderamiento y derechos de las mujeres en todo el mundo.

\section{Referencias bibliográficas}

\section{Libros y artículos}

Bergallo, Paola. 2014. The Struggle against Informal Rules on

Abortion in Argentina, Abortion Law in Rebecca J. Cook, Joanna

N. Erdman y Bernard M. Dickens (eds), Transnational

Perspective: Cases and Controversies.

Dembour, Marie Benedicte. 2018. Critiques, en International Human Rights Law, compilado por D. Moeckli, S. Shah, y S.

Sivakumaran. 3rd edn Oxford University Press.

Fine, Johanna B., Mayall, Katherine y Sepúlveda, Lilian. 2017. The Role of International Human Rights Norms in the Liberalization of Abortion Laws Globally, (El rol de las normas de derechos humanos en la liberalización de las leyes de aborto globalmente). Health and Human Rights Journal. Disponible en: https://cdn2.sph.harvard.edu/wpcontent/uploads/sites/125/2017/06/Fine.pdf 
Joyce, Molly. 2017. The Human Rights Aspects of Abortion. Hibernian L.J. 27.

Kälin, Walter y Künzli, Jorg. 2009. Notions and Sources, in The Law of International Human Rights Protection. Oxford.

Mitra, Anjori. 2017. We're Always Going to Argue about Abortion: International Law's Changing Attitudes towards Abortion. New Zealand Women's Law Journal.

Sedacca, Natalie. 2017. Abortion in Latin America in International Perspective: Limitations and Potentials of the Use of Human Rights Law to Challenge Restrictions (Aborto en América Latina en perspectiva internacional: limitaciones y potenciales en el uso del derecho de los derechos humanos para desafiar las restricciones). Berkeley J. Gender L. \& Just.

Pittier, Lautaro. 2016. Control de convencionalidad en Argentina. Corte Interamericana de Derechos Humanos. Disponible en: http://www.corteidh.or.cr/tablas/r36279.pdf

Pou Giménez, Francisca. 2019. La sentencia F.,A.L. y la despenalización por indicaciones: una encrucijada en el tratamiento jurídico del aborto en América Latina La Corte y sus presidencias en La Corte y sus presidencias, directoras Laura Clérico y Paula Gaido, coordinador Gustavo Arballo. Editorial Ad Hoc.

Ruibal, Alba y Fernandez Anderson, Cora. 2018. Legal obstacles and social change: strategies of the abortion rights movement in Argentina. Politics, Groups, and Identities (Obstáculos legales y cambio social: estrategias del movimiento de aborto legal en Argentina. Políticas, Grupos e Identidades).

Ruibal, Alba. 2015. Movilización y contra-movilización legal. Propuesta para su análisis en América Latina. Polít. gob vol. 22 no.1 México. Disponible en: http://www.scielo.org.mx/scielo.php?script=sci_arttext\&pid=S1 665-20372015000100006

Skuster, Patty. 2017. How laws fail the promise of medical abortion: a global look. 18 Geo. J. Gender \& L. 379.

Tomuschat, Christian. 2008. Between Idealism and Realism, Introduction. Oxford University Press. 
Constitución Nacional Argentina

Código Penal, Artículo 86.

Convención Interamericana Para Prevenir, Sancionar Y Erradicar La Violencia Contra La Mujer “Convención De Belem Do Para”, 9 de junio de 1994

Convención sobre la Eliminación de todas formas de Discriminación contra la Mujer, 18 de diciembre de 1979

Convención sobre los Derechos del Niño, 20 de noviembre de 1989

Declaración Universal de Derechos Humanos, 10 de diciembre de 1948

Protocolo para la Atención Integral de las Personas con Derecho a la Interrupción Legal del Embarazo (ILE):

http://www.msal.gob.ar/images/stories/bes/graficos/0000000875cn t-protocolo_ile_octubre\%202016.pdf

Jurisprudencia

Comisión Interamericana de Derechos Humanos

- Caso “Baby Boy”. Resolución Nro. 23/81, Caso 2141, Estados Unidos de América, Resolución del 6 de marzo de 1981

Corte Interamericana de Derechos Humanos

- Caso Artavia Murillo y otros (“Fecundación In Vitro") vs. Costa Rica (Excepciones Preliminares, Fondo, Reparaciones y Costas). Serie C No. 257. Sentencia de 28 de noviembre de 2012

Corte Suprema de Justicia de la Nación

- $\quad$ Expediente Letra “F,”. 'F., A. L.'. Serie No. 259, Libro XLVI, (Argentina), Sentencia de 13 de marzo de 2012

\section{Fuentes documentales}

Comité de Derechos Humanos. Observación General Nro. 36 sobre el artículo 6 del Pacto Internacional de Derechos Civiles y Políticos, relativo al derecho a la vida. CCPR/C/GC/36, 30 de octubre de 2018. Disponible en:

https:// www.ohchr.org/Documents/HRBodies/CCPR/GCArticl e6/GCArticle6_SP.pdf 
Comité de Derechos Humanos. Observación General Nro. 6, Artículo 6 - Derecho a la vida, $16^{\circ}$ período de sesiones, U.N. Doc. HRI/GEN/1/Rev.7 at 143 (1982). Disponible en: http://hrlibrary.umn.edu/hrcommittee/Sgencom6.html

Comité de Derechos Humanos. Observación General Nro. 14. El derecho a la vida (artículo 6). $23^{\circ}$ período de sesiones (1984). Disponible en:

https://tbinternet.ohchr.org/_layouts/15/treatybodyexternal/D ownload.aspx?symbolno=INT\%2fCCPR\%2fGEC\%2f4723\&Lang= es

Comité para la Eliminación de la Discriminación Contra la Mujer. Recomendación general Nro. 30 sobre las mujeres en la prevención de conflictos y en situaciones de conflicto y posteriores a conflictos, 1 de noviembre de 2013.

Comité de Derechos Económicos, Sociales y Culturales. Observación general Nro. 22 (2016), relativa al derecho a la salud sexual y reproductiva (artículo 12 del Pacto Internacional de Derechos Económicos, Sociales y Culturales), E/C.12/GC/22, 2 de mayo de 2016.

Comité de los Derechos Niño. Observación General No. 4, La salud y el desarrollo de los adolescentes en el contexto de la Convención sobre los Derechos del Niño, U.N. Doc. CRC/GC/2003/4 (2003). Disponible en: http://hrlibrary.umn.edu/crc/spanish/Sgeneralcomment4.html

Informe del Relator Especial de las Naciones Unidas sobre la Tortura y otros Tratos o Penas Crueles, Inhumanos o Degradantes. A/HRC/31/57, 5 de enero de 2016.

Informe del Relator Especial sobre el Derecho de Toda Persona al Disfrute del Más Alto Nivel Posible de Salud Física y Mental. A/HRC/32/32, 4 abril 2016.

Organización Mundial de la Salud, Constitución. Disponible en: http://apps.who.int/gb/bd/PDF/bd48/basic-documents-48thedition-sp.pdf\#page $=7$

Organización Mundial de la Salud. 2012. Aborto sin riesgos: guía técnica y de políticas para sistemas de salud. Disponible en:

https://apps.who.int/iris/bitstream/handle/10665/77079/978924354 8432_spa.pdf;jsessionid=2A10E72569B420634241E0B3A4B9AEC 5 ? sequence $=1$

Organización Mundial de la Salud. 2018. Manejo médico del aborto. 
Honorable Senado de la Nación Argentina. Sitio oficial. Proyecto de ley de aborto. 2018. Disponible en:

https://www.senado.gov.ar/micrositios/ive/4

Cámara de la Honorable Diputados. Proyecto de Ley de Interrupción Voluntaria del Embarazo (IVE). Sitio oficial:

https://www.hcdn.gob.ar/export/hcdn/prensa/PDF/PROYECTO_D E_LEY_INTERRUPCIXN_VOLUNTARIA_DEL_EMBARAZO_2810D-2019.pdf

\section{Diarios}

Clarín, 2019a. “Tucumán: aseguraron que iban a interrumpir el embarazo de la nena de 11 años, pero le hicieron una cesárea”, 27 de febrero de 2019. Disponible en:

https://www.clarin.com/sociedad/tucuman-aseguraron-ibaninterrumpir-embarazo-nena-11-anos-hicieron-cesarea_0_X0EwOuwK.html

Clarín, 2019b. “Aborto: qué cambios propone el nuevo protocolo de interrupción legal del embarazo“, 20 de noviembre de 2019. Más información en: https://www.clarin.com/sociedad/abortocambia-nuevo-protocolo-interrupcion-legalembarazo_0_JfGE6y3S.html

Clarín, 2019c. "El Gobierno oficializó la renuncia de Adolfo Rubinstein en Salud", 26 de noviembre de 2019. Más información en: https://www.clarin.com/politica/gobiernooficializo-renuncia-adolfo-rubinstein-ministeriosalud_0_aEkklrOl.html

Clarín 2019d. “Cómo es el nuevo protocolo de aborto no punible” 13 de diciembre de 2019. Disponible en:

https://www.lanacion.com.ar/sociedad/como-es-nuevoprotocolo-aborto-no-punible-nid2315497

El Cronista, 2019. “Entró en vigencia el nuevo protocolo del aborto no punible: en qué consiste", 13 de diciembre de 2019, Disponible en: https://www.cronista.com/economiapolitica/ Entro-en-vigencia-el-nuevo-protocolo-del-aborto-no-punibleen-que-consiste-20191213-

0002.html?utm_source=ecc_nota\&utm_medium=cms\&utm_ca mpaign=refresh 
El País, 2019. "El aborto se abre paso en la campaña electoral argentina", 29 de mayo de 2019. Disponible en:

https://elpais.com/sociedad/2019/05/28/actualidad/155906237 1_230742.html

La Nación, 2019. “Niñas madres con mayúsculas”. 1 de febrero de 2019. Disponible en:

https://www.lanacion.com.ar/opinion/ninas-madres-conmayusculas-nid2216199

Página 12, "El derecho de la niña a no ser madre", 26 de febrero de 2019. Disponible en: https://www.pagina12.com.ar/177346-elderecho-de-la-nina-a-no-ser-madre

\section{Sitios Web}

Campaña Nacional por el Derecho al Aborto Legal, Seguro y Gratuito, sitio oficial. http://www.abortolegal.com.ar/about/

Campaña Nacional por el Derecho al Aborto Legal, Seguro y Gratuito. http://www.abortolegal.com.ar/proyecto-de-ley-delegalizaciondespenalizacion-del-aborto-en-argentina/

CELS, Informe conjunto remitido al Comité de Derechos Económicos, Sociales y Culturales (comité DESC) de las Naciones Unidas para la cuarta evaluación periódica del país. 2018. https://www.cels.org.ar/web/wpcontent/uploads/2018/09/20180911_Aborto_ComiteDESC.pdf

CELS, Aborto legal: argumentos, legislación y jurisprudencia. Publicación del Centro de Estudios Legales y Sociales. 2018a. https://www.cels.org.ar/web/wpcontent/uploads/2018/05/AbortoLegal.pdf

Equipo Latinoamericano de justicia y género (ELA), Red de acceso al aborto seguro - Argentina (REDAAS) y Centro de Estudios de Estado y Sociedad (CEDES). 2018b. Las cifras del aborto en Argentina. http://www.redaas.org.ar/archivos-actividades/64CIFRAS\%20ABORTO-REDAAS-singlepage.pdf

Equipo Latinoamericano de justicia y género (ELA), Red de acceso al aborto seguro - Argentina (REDAAS) y Centro de Estudios de Estado y Sociedad (CEDES). 2018.

Monitoreo del debate sobre legalización del aborto en Argentina Informe 1: 
http://www.ela.org.ar/a2/index.cfm?muestra\&codcontenido=3 $087 \&$ plcontampl $=43 \&$ aplicacion $=$ app $187 \& \mathrm{cnl}=15 \&$ opc $=49$

Monitoreo del debate sobre legalización del aborto en Argentina Informe 2: http://www.redaas.org.ar/archivos-actividades/90Monitoreo\%20del\%20debate\%20sobre\%20aborto\%20\%20Informe\%202.pdf

Encuentro Nacional de Mujeres, sitio oficial. http://encuentrodemujeres.com.ar/

Ni Una Menos, sitio oficial. http://niunamenos.org.ar/quienessomos/carta-organica/

ONU Mujeres, nociones básicas sobre incorporación de la perspectiva de género. https://www.un.org/womenwatch/osagi/gendermainstreaming .htm 